\title{
Detection of Coronary Atherosclerotic Plaques with Superficial Proteoglycans and Foam Cells using Real-Time Intrinsic Fluorescence Spectroscopy
}

\author{
George O. Angheloiu, M.D. , Abigail S. Haka, Ph.D.., Irene Georgakoudi, Ph.D.*, Joseph \\ Arendt, Ph.D. , Markus G. Müller, Ph.D. , Obrad R. Scepanovic, Ph.D. , Stephen P. Evanko, \\ Ph.D.ף, Thomas N. Wight, Ph.D. đ, Prasun Mukherjee, Ph.D.§, David H. Waldeck, Ph.D.§, \\ Ramachandra R. Dasari, Ph.D. ${ }^{*}$ Maryann Fitzmaurice, M.D., Ph.D. ${ }^{\star}$, , John R. Kramer, \\ M.D. , and Michael S. Feld, Ph.D. \\ "Spectroscopy Laboratory, Massachusetts Institute of Technology, Cambridge, MA \\ ${ }^{\dagger}$ Case Western Reserve University and University Hospitals-Case Medical Center, Cleveland, $\mathrm{OH}$ \\ "Benaroya Research Institute, Seattle, WA \\ $\S$ Department of Chemistry, University of Pittsburgh, Pittsburgh, PA
}

\begin{abstract}
Objectives-The protein components of low-density lipoprotein (LDL), oxidized LDL and proteoglycans such as versican contain tryptophan, an amino acid with characteristic fluorescence features at $308 \mathrm{~nm}$ excitation wavelength. We hypothesize that intrinsic fluorescence spectroscopy at $308 \mathrm{~nm}$ excitation wavelength (IFS 308 ), a method suitable for clinical use, can identify coronary artery lesions with superficial foam cells (SFCs) and/or proteoglycans.
\end{abstract}

\begin{abstract}
Methods-We subjected 119 human coronary artery specimens to in vitro fluorescence and reflectance spectroscopy. We used 5 basis spectra to model $\mathrm{IFS}_{308}$, and extracted their contributions to each individual IFS ${ }_{308}$ spectrum. A diagnostic algorithm using the contributions of total tryptophan and fibrous cap to IFS 308 was built to identify specimens with SFCs and/or proteoglycans in their top $50 \mu \mathrm{m}$.
\end{abstract}

Results-We detected SFCs and/or proteoglycans, such as versican or the glycosaminoglycan hyaluronan, in 24 fibrous cap atheromas or pathologic intimal thickening (PIT) lesions. An algorithm using the contributions of total tryptophan and fibrous cap to $\mathrm{IFS}_{308}$ was able to identify these segments with $92 \%$ sensitivity and $80 \%$ specificity.

Conclusion-We were able to establish a set of characteristic LDL, oxidized LDL, versican and hyaluronan fluorescence spectra, ready to be used for real-time diagnosis. The IFS 308 technique detects SFCs and/or proteoglycans in fibrous cap atheromas and PIT lesions. SFCs and

(C) 2010 Elsevier Ireland Ltd. All rights reserved.

Corresponding author: George O. Angheloiu, M.D., 209 Hospital Ave., Dubois, PA 15801, Telephone: 814591 7031, Fax: 617253 4513, goangheloiu@drmc.org.

In memory of Michael, our teacher.

Publisher's Disclaimer: This is a PDF file of an unedited manuscript that has been accepted for publication. As a service to our customers we are providing this early version of the manuscript. The manuscript will undergo copyediting, typesetting, and review of the resulting proof before it is published in its final citable form. Please note that during the production process errors may be discovered which could affect the content, and all legal disclaimers that apply to the journal pertain.

There are no conflicts of interest for any of the authors listed. 
proteoglycans are histological markers of vulnerable plaques, and this study is a step further in developing an invasive clinical tool to detect the vulnerable atherosclerotic plaque.

\section{Keywords}

tryptophan; low-density lipoprotein; versican; foam cells; spectroscopy

\section{Introduction}

Thrombosis of rupture- or erosion-prone vulnerable atherosclerotic plaques is believed to be the underlying cause of most acute coronary events [1]. Attention has been focused on the role played by lipoproteins, lipid-laden macrophages, and the extracellular matrix in the process of plaque erosion [2]. Matrix proteoglycans serve as scaffolding that enables accumulation of low-density lipoprotein (LDL), migration of smooth muscle cell and accumulation of lipoprotein-laden foam cells into the plaque [3-5].

Apo (a) and apo B100 and proteoglycans such as versican and decorin-biglycan contain tryptophan in their protein structure [6-9]. In contrast, elastin, collagen I and collagen III, the most important structural proteins of normal artery and stable plaques, do not harbor tryptophan in their structure (www.uniprot.org).

Our group has previously detected pathognomonic fluorescence features indicating the presence of tryptophan in atherosclerotic artery specimens using $310 \mathrm{~nm}$ excitation light [10]. We hypothesize that by quantifying the fluorescence imprint of tryptophan with intrinsic fluorescence spectroscopy (IFS), a method devised by our laboratory [11], the presence of lipoprotein-rich superficial foam cells (SFCs) and proteoglycans could be detected, allowing the identification of plaques prone to erosion or rupture.

Our prior work has demonstrated a method useful in the identification of SFCs, using data generated by diffuse reflectance spectroscopy (DRS) and IFS at $480 \mathrm{~nm}$ excitation [12]. Interestingly the $480 \mathrm{~nm}$ excitation wavelength was not useful for the identification of proteoglycans [12]. The current investigation advances our results by adding a new element in the vulnerable plaque equation: the global detection of proteoglycans and SFCs.

\section{Methods}

\section{Subjects and Specimens}

This study was approved by the Massachusetts Institute of Technology Committee on the Use of Humans as Experimental Subjects and the Cleveland Clinic Foundation's Institutional Review Board, in consultation with the Cuyahoga County Coroner's Office. 119 coronary artery specimens were harvested from autopsy cases $(n=11)$ and hearts explanted during cardiac transplant operations $(n=3)$ [13]. The average age of the 14 subjects was 59 and 9 of them were male. Coronary artery disease was the cause of death in 6 of the autopsy cases and sudden cardiac death was documented in 5. The 3 transplant recipients had a clinical diagnosis of ischemic cardiomyopathy.

Specimens were handled and prepared for spectroscopy measurements as previously detailed [12]. They were used after snap-freezing and thawing. Normalized $308 \mathrm{~nm}$ excitation intrinsic fluorescence ( $\mathrm{IFS}_{308}$ ) as well as DRS data acquired prior to $-80^{\circ} \mathrm{C}$ freezing and after thawing were proven to be consistent (online Figure 1-supplemental). 


\section{IFS and DRS Measurements}

Instrumentation-To collect fluorescence and DRS data required to obtain the intrinsic fluorescence spectra, we used a FastEEM instrument developed in our laboratory. This unit is equipped with a fiber optic probe for light delivery and collection and has already been used successfully in clinical studies [14]. The instrument, optical theory, specimen preparation and calibration methods have been described in prior work and detailed in the online supplemental file theory_instrumentation_supplemental_file.doc [12].

IFS $_{308}$ Basis Spectra-IFS 308 was obtained using the DRS and $308 \mathrm{~nm}$ fluorescence data as previously described [12]. Based on our previous experience, a candidate set of basis spectra was established, and then narrowed to a minimal number of spectra that fit the coronary spectra with maximum accuracy via non-negativity constrained least-squares minimization. The 6 initial candidate basis spectra were: fibrous cap (FCAP), media (MED), tryptophan (TRP), red-shifted (RS), LDL spectrum, and calcification spectrum. FCAP, MED, and calcification spectra were obtained by averaging the IFS 308 spectra of sets of 5 layers of each individual type of coronary tissue. LDL was extracted from human plasma, and tryptophan was purchased from Sigma-Aldrich, St. Louis, MO. The RS spectrum was obtained by multiple curve resolution analysis of a set of 5 whole coronary specimens with a large amount of SFCs in the top layers, as previously described [12].

\section{Identification of Tryptophan Fluorescence in LDL and Proteoglycans Spectra}

-We hypothesized that spectra of LDL and two families of proteoglycans mostly encountered in coronary atherosclerotic lesions (versican and decorin-biglycan) demonstrate signs of tryptophan fluorescence. For this reason we obtained spectra of two vascular proteoglycan preparations: versican and decorin-biglycan, which were isolated and purified by methods described by Hascall et al with modifications [15]. Hyaluronan extracted from bovine vitreous humor was purchased from Sigma-Aldrich, St. Louis, MO. To document what proteoglycan spectra demonstrate spectroscopic features of tryptophan, we modeled them with the help of the basis spectra selected to fit the coronary specimens' spectroscopic data. With the purpose of a more accurate definition of the hyaluronan fluorescence we obtained spectra of oligo-4 hyaluroanan (Hyalose, Oklahoma City, OK) and of its two monomers, N-acetyl-D-glucosamine (NAG) and D-glucuronic acid (Sigma, St. Louis, MO) as described in the online file theory_instrumentation_supplemental_file.doc.

In order to demonstrate that tryptophan is contributing to the LDL fluorescence, we compared spectra of tryptophan, LDL and of various oxidized LDL (OxdLDL) species generated according to a oxidation method described elsewhere [20]. During the oxidation process spectra were acquired every 1-2 hours for the first 24 hours and then every 24 hours for up to 5 days. Background for the spectroscopic measurements was acquired from a $\mathrm{CuSO}_{4}$ solution as used in the oxidation method quoted [16].

IFS $_{\mathbf{3 0 8}}$ Modeling-IFS 308 data were modeled by a linear combination of a minimum number of $\mathrm{IFS}_{308}$ basis spectra that would provide the best fit [12], which allowed us to extract the relative fit contributions of the basis spectra to the experimental IFS 308 . Goodness of fit was measured by root mean square error of the residual (experimental spectrum minus model fit) divided by peak $\mathrm{IFS}_{308}$.

While modeling by way of a minimum number of basis spectra was aimed at providing the best possible and most robust fit, this processing step did not necessarily demonstrate the atherosclerotic plaque-specific molecules targeted by us, such as LDL, oxidized LDL (OxdLDL), versican, hyaluronan and decorin-biglycan, contributed to IFS 308 spectra of the 
specimens analyzed. With this objective in mind we used these spectra to perform a second modeling run.

\section{Histology}

Coronary segments were routinely processed and cut into serial $5 \mu \mathrm{m}$-thick sections. All specimens were subjected to hematoxylin-eosin and Movat's pentachrome staining for identification of proteoglycans (blue), collagen (yellow) and elastin fibers (black). Intense proteoglycan staining was defined as intense blue coloration on Movat's pentachrome in the absence of elastin or collagen fibers. Foam cells were identified histologically by their foamy cytoplasm. Specimens with either SFCs or superficial proteoglycan accumulations, alone or in combination, were subjected to immunohistochemical studies for the identification of versican (anti-versican antisera - LF99), kindly provided by Dr. Larry Fisher (Craniofacial and Skeletal Disease Branch, National Institutes of Dental Research, $\mathrm{NIH}$, Bethesda, MD) and hyaluronan (hyaluronan binding protein, gift of Dr. Charles Underhill, Georgetown University, Washington, DC) $[17,18]$. The purpose of the latter two staining methods was to demonstrate that each of the diagnostic group's specimens harbored SFCs and/or species of proteoglycans known to predispose the coronary plaque to the process of erosion.

Slides were examined by a cardiovascular pathologist blinded to the spectroscopic results who used a modified American Heart Association classification [19], dividing the coronary segments into: normal, intimal thickening, intimal xanthoma, pathological intimal thickening, fibrous cap atheroma, fibrocalcific plaque, and thin fibrous cap atheroma (cap thickness $<65 \mu \mathrm{m}$ ). Proteoglycans and SFCs were identified on each slide in the region of interest to a depth of $50 \mu \mathrm{m}$, which corresponds to the sampling depth at $308 \mathrm{~nm}$ excitation [20].

The diagnostic target group consisted of three subgroups: specimens with SFCs alone, a combination of SFCs and superficial proteoglycans, or intense proteoglycan staining at the same time positive for hyaluronan or versican in the absence of SFCs.

\section{Analysis Methods and Diagnostic Algorithms}

The purpose of our study was the identification of the diagnostic target group defined above. To achieve this goal, we employed an algorithm using as diagnostic variables the basis spectra contributions to IFS $\mathrm{F}_{308}$. To analyze the data set in an unbiased manner we used logistic regression and leave-one-out cross-validation, method that obviates the need for a training and validation set of [12].

\section{Results Histology}

We investigated 119 coronary specimens: 32 normal and intimal thickening lesions, 4 intimal xanthomas, 23 pathological intimal thickening, 45 fibrous cap atheromas, 0 thin fibrous cap atheromas, and 15 fibrocalcific plaques.

SFCs alone were identified in 4 coronary specimens. Fifteen coronary specimens demonstrated SFCs accompanied by proteoglycans in the same region. In 5 other coronary specimens, we identified intense proteoglycan staining by Movat's pentachrome accompanied by positive immunohistochemical staining for hyaluronan or versican, in the absence of SFCs. The combination of these three subgroups represented our diagnostic algorithm's target group $(n=24)$. 
Tissue was available for anti-versican and hyaluronan binding protein staining in 20 and 18 specimens of the diagnostic target group. The two staining modalities were positive in 18 and 14 of these specimens (90\% and 78\% respectively).

\section{IFS and DRS Measurements}

IFS $_{\mathbf{3 0 8}}$ Basis Spectra-The 6 candidate IFS S $_{308}$ basis spectra were listed in the Methods section. Only five of them were required for optimal model fits: FCAP, MED, TRP, LDL, and RS (Figure 1a). The use of each of these basis spectra resulted in significantly better fits than the models performed in their absence $(\mathrm{P}<0.05)$. The use of the calcification spectrum did not make a difference in the $\mathrm{IFS}_{308}$ fit $(\mathrm{P}=0.85)$.

FCAP and MED basis spectra were similar to those of collagen and elastin (Figure 1b) (peaks at $401 \mathrm{~nm}, 413,405 \mathrm{~nm} 406 \mathrm{~nm}$ respectively). The fits were significantly better using FCAP and MED than using collagen and elastin ( $\mathrm{P}=0.02$ for goodness of fit).

\section{Identification of Tryptophan Fluorescence in LDL and Proteoglycans Spectra}

-The LDL basis spectrum peaked at $348 \mathrm{~nm}$, demonstrating a very large shift towards the ultra-violet spectral region in comparison with FCAP and MED, while the tryptophan spectrum peaked at $362 \mathrm{~nm}$. Despite the $14 \mathrm{~nm}$ shift between the LDL and tryptophan peaks, we suspected that the LDL fluorescence is due to tryptophan. To verify this assertion, we acquired spectra of LDL oxidized using the $\mathrm{Cu}^{+2}$ method [16]. At 1 hour the OxdLDL spectrum had a peak in the same region $(363 \mathrm{~nm})$ with that of TRP $(362 \mathrm{~nm})$ (Figure 1c). At 2 hours the peak in the $348-362 \mathrm{~nm}$ region disappeared, making room for a peak situated at $430 \mathrm{~nm}$ (online Figure 2-supplemental).

Figure 2 illustrates the spectra of versican, decorin-biglycan, OxdLDL, and hyaluronan and their fits using our 5 basis spectra. As expected their modeling demonstrated a relatively high contribution of tryptophan to versican, decorin-biglycan and OxdLDL. Hyaluronan demonstrated a significant contribution from the tryptophan spectrum as well (Figure 2). Since tryptophan is not a structural component of hyaluronan, we acquired spectra of oligo-4 hyaluronan, and its two monomers NAG and D-glucuronic acid. Figure 3-suplemental demonstrates that both oligo-4 hyaluronan and NAG have peaks in the near-UV region, at $362 \mathrm{~nm}$ and $348 \mathrm{~nm}$ respectively. This suggests that NAG is the chemical moiety responsible for the tryptophan pseudo-contribution to the hyaluronan spectrum.

IFS $_{\mathbf{3 0 8}}$ modeling-In order to extract data regarding the contribution of LDL and TRP to IFS $_{308}$ we used a modeling system employing the minimum number of basis spectra required to obtain an excellent fit: FCAP, MED, TRP, LDL, and RS (Figure 1a). We calculated a diagnostic variable labeled Total Tryptophan as the sum of LDL and TRP contributions. Total Tryptophan contribution, defined in this manner, represents the expression of the major trytophan-containing molecules in the atherosclerotic plaques: LDL, OxdLDL, versican and decorin-biglycan. A chemical moiety with fluorescence similar with tryptophan is NAG (Figure 3-suplemental) - a structural component of hyaluronan and biglycan [21], not known to be present in collagen, elastin, versican and LDL. Since its fluorescence properties at $308 \mathrm{~nm}$ excitation are similar to tryptophan, we considered for reasons of diagnostic simplicity to include its fluorescence contribution in the Total Tryptophan contribution.

The Total Tryptophan burden may include the participation of other tryptophan-containing molecules as well, such as the matrix regulatory proteins (osteopontin and osteocalcin) or matrix metalloprotease proteins, which are known to contain tryptophan (http://www.uniprot.org). Figure 3 illustrates the spectra of osteopontin (Sigma-Aldrich, St. Louis, MO) and osteocalcin (graciously donated by Dr. Caren Gundberg, Yale University, 
New Haven, CT) [22]. One can notice their spectral peaks stretching from $368 \mathrm{~nm}$ to 374 $\mathrm{nm}$, close to the $361 \mathrm{~nm}$ location of the tryptophan peak, suggesting a significant spectral contribution from this amino acid.

Overall, the average Total Tryptophan contribution was $28 \% \pm 19 \%$ in the diagnostic target group $(\mathrm{n}=24)$, much higher than $6 \% \pm 8 \%$ for the coronary lesions without SFCs or proteoglycan deposits ( $\mathrm{n}=95)$ (Figure 4$)$. The highest Total Tryptophan contribution was $65 \%$, recorded in a sample with abundant superficial SFCs and proteoglycans.

IFS $_{308}$ modeling using spectra of LDL and proteoglycans-In order to demonstrate that LDL and proteoglycans were indeed the tryptophan-containing chemical moieties contributing to $\mathrm{IFS}_{308}$, we performed a second modeling of our diagnostic target group using the actual spectra of LDL, versican, hyaluronan, decorin-biglycan and FCAP. The fits were only slightly inferior in accuracy to those obtained by way of the 5 basis spectra (goodness of fit $97.4 \%$ versus $98.2 \%, \mathrm{P}<0.05$ ). Average contributions of LDL and proteoglycans were $15 \%$ and $19 \%$. Contributions of versican, hyaluronan, and decorinbiglycan were $7 \%, 3 \%$ and $9 \%$ respectively.

\section{Diagnostic algorithm}

A binary diagnostic algorithm using Total Tryptophan contribution and FCAP contribution demonstrated a sensitivity of $92 \%$, specificity of $80 \%$ and total accuracy of $82 \%$ (Figure 5) in identifying the diagnostic target group (total $n=24$ ).

\section{Discussion}

Heart disease remains the leading cause of death in both men and women in the United States. Plaque rupture and plaque erosion account for most cases of myocardial infarction and sudden death [1].

The current study demonstrates that intrinsic fluorescence is able to collectively identify proteoglycans and SFCs in coronary specimens. Proteoglycans, LDL and SFCs are plaque elements that co-localize and functionally interact very closely, contributing to the development of plaque erosion or rupture [3,23]. Superficial lipoprotein-rich macrophages are a landmark pathological feature of ruptured lesions, and are present in the eroded plaques as well, representing $3.44 \%$ versus $2.53 \%$ of the intimal surface in ruptured and eroded plaques respectively [1,24]. Proteoglycans, of which versican and the glycosaminoglycan hyaluronan are the most important for the purpose of vulnerable plaque identification [25], are found both in the thick fibrous cap atheromas and pathological intimal thickening lesions, as well as in the lipid-rich core of fibrous cap atheromas [23]. Therefore the identification of proteoglycans, lipoproteins and foam cells as a group would represent an important step in developing a spectroscopic clinical tool for the detection of vulnerable atherosclerotic plaques.

\section{Spectroscopic principle of global lipoprotein and proteoglycan identification}

Our method is based on the fluorescence properties of tryptophan. The molecules we investigated were those related to plaque vulnerability: LDL, OxdLDL and proteoglycans. The LDL spectrum had a shape very similar to that of tryptophan, with a peak situated at $348 \mathrm{~nm}, 14 \mathrm{~nm}$ away from the tryptophan peak (Figure 1a). The spectrum of a molecule similar to LDL - LDL oxidized for 1 hour using the $\mathrm{Cu}^{+2}$ method (OxdLDL) - displayed a peak superimposed with the tryptophan peak, offering a second reason to believe that the near-ultraviolet LDL fluorescence is due to tryptophan (Figure 1c). The 2-hour oxidation product of the same reaction lost the fluorescence peak in the $348-362 \mathrm{~nm}$ region 
characteristic for tryptophan (online Figure 2-supplemental). In agreement with our data, numerous reports have demonstrated the concomitant loss of tryptophan residues and tryptophan fluorescence during the $\mathrm{Cu}^{+2}$ oxidation of LDL [26]. This is a third argument to indicate that the fluorescence peak in the 348-362 nm region of LDL and 1-hour oxidized LDL is due to tryptophan.

We also demonstrated that versican, a proteoglycan with an important role in the process of coronary erosion, has features of tryptophan fluorescence. Its spectrum showed a tryptophan contribution of 57\%, and a peak situated very closely to that of tryptophan (Figure 2).

Our hyaluronan samples demonstrated features of tryptophan fluorescence as well (34\% tryptophan spectra contribution), in spite of the fact that hyaluronan's structure does not include tryptophan. To elucidate the origin of these features we acquired spectra of oligo-4 hyaluronan. This is a small hyaluronan molecule synthesized through an enzymatic process, which excludes the possibility of tryptophan contamination from outside sources. The latter can occur in the large hyaluronan molecules extracted from animal tissue, where tryptophancontaining versican moieties could still be linked onto the hyaluronan chain through the intermediation of hyaluronan binding domains [26]. Interestingly though, in a similar way with the large hyaluronan, oligo-4 hyaluronan showed a fluorescence feature with a peak at $362 \mathrm{~nm}$ (online Figure 3-supplemental). Spectra of the two monomers, NAG and Dglucuronic acid, were acquired as well. NAG demonstrated a peak at $348 \mathrm{~nm}$, closely located to tryptophan and oligo-4 hyaluronan, which can explain the fluorescence features of hyaluronan in this spectral region. NAG fluorescence properties are similar to tryptophan, and we considered for reasons of diagnostic simplicity to include its fluorescence contribution in the Total Tryptophan contribution.

Since LDL, OxdLDL, versican and hyaluronan have strong contributions from tryptophan and NAG, we designed a global parameter labeled Total Tryptophan that includes the contributions of tryptophan, LDL and for reasons of simplicity NAG as well.

Hyaluronan is an important component in the superficial layers of erosion-prone plaques and it is known to aggregate with smaller tryptophan-containing molecules such as versican and lipoproteins $[3,25,28]$. Thus, in addition to its fluorescence properties derived from NAG, we hypothesize that hyaluronan can de indirectly detected as well by identifying the areas rich in tryptophan-containing versican and lipoproteins.

Matrix metalloproteases and regulatory proteins (osteopontin and osteocalcin) are important molecules in the plaque physiology, and they are known to contain tryptophan in their structure - as suggested spectroscopically by Figure 3. A parameter such as Total Tryptophan would incorporate the presence of these molecules as well, which could represent an important feature of this global diagnostic parameter. Further work is needed for the individual identification of these proteins. The online Supplemental Table is a more comprehensive list of tryptophan-containing proteins and protein-including structures pertaining to the lesions studied in this report.

Importantly, the collagen and elastin chains - the main chemical constituents of FCAP, MED and stable plaques - do not contain tryptophan or NAG. This is clearly demonstrated by their spectral peaks ( $405 \mathrm{~nm}$ and $406 \mathrm{~nm}$ ) being situated at a large distance from the tryptophan $(362 \mathrm{~nm})$ and tryptophan-containing molecules. Since stable plaques contain mostly collagen and elastin (found in the fibrous cap and media layers) while unstable plaques are rich in tryptophan-containing molecules such as proteoglycans, LDL and OxdLDL, the spectral differences described here could allow for an accurate differentiation between the stable and unstable plaques. Our results add further evidence to a growing body of 
spectroscopy literature searching for the best invasive method of vulnerable plaque identification $[29,30,31]$.

\section{Histological evidence of lipoproteins, versican and hyaluronan}

Our target diagnostic group was formed by specimens with accumulations of SFCs and proteoglycans in their superficial layers $(n=24)$. Since lipoproteins (in our case represented by their cellular surrogate - SFCs), versican and hyaluronan are main chemical moieties of the vulnerable plaque, we felt necessary to demonstrate that they were present in the majority of the target group specimens. Indeed these three histological elements were found respectively in $79 \%, 90 \%$ and $78 \%$ of the specimens investigated, and at least one of them was found in each specimen of the diagnostic group.

\section{Diagnostic potential}

We established in this study a set of intrinsic fluoresce spectra of high medical interest, which were used to identify coronary plaques with superficial proteoglycans and foam cells. Importantly, intrinsic fluorescence spectra can be acquired in less than $200 \mathrm{~ms}$ and analyzed online in less than 1 second, allowing for real-time chemical quantitative analysis. In addition, the fiberoptic probe is approximately 3 meters in length and $1 \mathrm{~mm}$ thick allowing to be inserted through a catheter into the coronary vessels in order to acquire spectra of coronary plaques.

\section{Limitations}

Our group of coronary specimens did not include thin fibrous cap atheromas, arterial thrombi or intra-plaque hemorrhages, and thus we could not characterize the role of tryptophan fluorescence in these types of lesions.

\section{Conclusion}

We were able to establish a set of characteristic LDL, oxidized LDL and versican fluorescence spectra, ready to be used for real-time diagnosis. IFS 308 had the ability to identify coronary plaques with SFCs and/or proteoglycans in their superficial $50 \mu \mathrm{m}$. These are features of plaques prone to erosion or rupture, identification of which is an important step in developing a fiber-optic invasive clinical tool to detect vulnerable atherosclerotic plaques.

\section{Supplementary Material}

Refer to Web version on PubMed Central for supplementary material.

\section{Acknowledgments}

We are indebted for invaluable technical support and research counseling to Dr. Vincent Hascall, Dr. Eugene Podrez, Lerner Research Institute, and Dr. Christine Moravec, Kaufman Center for Heart Failure, Cleveland Clinic, Cleveland, $\mathrm{OH}$.

This work was supported by funds from Pfizer, Inc, and the MIT Laser Biomedical Research Center, as well as National Institutes of Health grants RR02594 and R01 HL64675.

\section{References}

1. Kramer MC, Rittersma SZ, Winter RJ, et al. Relationship of Thrombus Healing to Underlying Plaque Morphology in Sudden Coronary Death. J Am Coll Cardiol 2010;55:122-132. [PubMed: 19818571] 
2. Farb A, Burke AP, Tang AL, et al. Coronary plaque erosion without rupture into a lipid core. A frequent cause of coronary thrombosis in sudden coronary death. Circulation 1996;93:1354-1363. [PubMed: 8641024]

3. Williams KJ, Tabas I. The response-to-retention hypothesis of early atherogenesis. Arterioscler Thromb Vasc Biol 1995;15:551-561. [PubMed: 7749869]

4. Wight TN, Merrilees MJ. Proteoglycans in atherosclerosis and restenosis: key roles for versican. Circ Res 2004;94:1158-1167. [PubMed: 15142969]

5. Gutierrez P, O'Brien KD, Ferguson M, Nikkari ST, Alpers CE, Wight TN. Differences in the distribution of versican, decorin, and biglycan in atherosclerotic human coronary arteries. Cardiovasc Pathol 1997;6:271-278.

6. Marcovina SM, Morrisett JD. Structure and metabolism of lipoprotein (a). Curr Opin Lipidol 1995;6:136-145. [PubMed: 7648002]

7. Borén J, Ekström U, Agren B, et al. The molecular mechanism for the genetic disorder familial defective apolipoprotein B100. J Biol Chem 2001;276:9214-9218. [PubMed: 11115503]

8. Zimmermann DR, Ruoslahti E. Multiple domains of the large fibroblast proteoglycan, versican. EMBO J 1989;8:2975-2981. [PubMed: 2583089]

9. Fisher LW, Termine JD, Young MF. Deduced protein sequence of bone small proteoglycan I biglycan) shows homology with proteoglycan II (decorin) and several nonconnective tissue proteins in a variety of species. J Biol Chem 1989;264:4571-4576. [PubMed: 2647739]

10. Baraga JJ, Rava RP, Fitzmaurice M, et al. Characterization of the fluorescent morphological structures in human arterial wall using ultraviolet-excited microspectrofluorimetry. Atherosclerosis 1991;88:1-14. [PubMed: 1878005]

11. Zhang Q, Müller MG, Wu J, Feld MS. Turbidity-free fluorescence spectroscopy of biological tissue. Opt. Lett 2000;25:1451-1453. [PubMed: 18066245]

12. Angheloiu GO, Arendt JT, Müller MG, et al. Intrinsic fluorescence and diffuse reflectance spectroscopy identify superficial foam cells in coronary plaques prone to erosion. Arterioscler Thromb Vasc Biol 2006;26:1594-1600. [PubMed: 16675721]

13. Sprung J, Ogletree-Hughes ML, Moravec CS. The effects of etomidate on the contractility of failing and nonfailing human heart muscle. Anesth Analg 2000;91:68-75. [PubMed: 10866889]

14. Georgakoudi I, Sheets EE, Muller MG, et al. Trimodal spectroscopy for the detection and characterization of cervical precancers in vivo. Am J Obstet Gynecol 2002;186:374-382. [PubMed: 11904594]

15. Hascall VC, Calabro A, Midura RJ, Yanagishita M. Isolation and characterization of proteoglycans. Methods Enzymol 1994;230:390-417. [PubMed: 8139509]

16. Podrez EA, Hoppe G, O'Neil J, et al. Macrophage receptors responsible for distinct recognition of low-density lipoprotein containing pyrrole or pyridinium adducts: models of oxidized low-density lipoprotein. J Lipid Res 2000;41:1455-1463. [PubMed: 10974053]

17. Bernstein EF, Fisher LW, Li K, et al. Differential expression of the versican and decorin genes in photoaged and sun-protected skin. Comparison by immunohistochemical and northern analyses. Lab Invest 1995;72:662-669. [PubMed: 7783424]

18. Green SJ, Tarone G, Underhill CB. Distribution of hyaluronate and hyaluronate receptors in the adult lung. J Cell Sci 1988;89:145-156. [PubMed: 2461953]

19. Virmani R, Kolodgie FD, Burke AP, et al. Lessons from sudden coronary death: a comprehensive morphological classification scheme for atherosclerotic lesions. Arterioscler Thromb Vasc Biol 2000;20:1262-1275. [PubMed: 10807742]

20. Sćepanović OR, Fitzmaurice M, Gardecki JA, et al. Detection of morphological markers of vulnerable atherosclerotic plaque using multimodal spectroscopy. J Biomed Opt 2006;11 021007.

21. Scott PG, Dodd CM, Bergmann EM, et al. Crystal structure of the biglycan dimer and evidence that dimerization is essential for folding and stability of class I small leucine-rich repeat proteoglycans. J Biol Chem 2006;281:13324-13332. [PubMed: 16547006]

22. Gundberg CM, Hauschka PV, Lian JB, et al. Osteocalcin: isolation, characterization, and detection. Methods Enzymol 1984;107:516-544. [PubMed: 6094965] 
23. Evanko SP, Raines EW, Ross R, et al. Proteoglycan distribution in lesions of atherosclerosis depends on lesion severity, structural characteristics, and the proximity of platelet-derived growth factor and transforming growth factor-beta. Am J Pathol 1998;152:533-546. [PubMed: 9466580]

24. van der Wal AC, Becker AE, van der Loos CM, et al. Site of intimal rupture or erosion of thrombosed coronary atherosclerotic plaques is characterized by an inflammatory process irrespective of the dominant plaque morphology. Circulation 1994;89:36-44. [PubMed: 8281670]

25. Kolodgie FD, Burke AP, Farb A, et al. Differential accumulation of proteoglycans and hyaluronan in culprit lesions: insights into plaque erosion. Arterioscler Thromb VascBiol 2002;22:1642-1648.

26. Giessauf A, Steiner E, Esterbauer H. Early destruction of tryptophan residues of apolipoprotein B is a vitamin E-independent process during copper-mediated oxidation of LDL. Biochim Biophys Acta 1995;1256:221-232. [PubMed: 7766701]

27. Ohno-Jinno A, Isogai Z, Yoneda M, et al. Versican and fibrillin-1 form a major hyaluronanbinding complex in the ciliary body. Invest Ophthalmol Vis Sci 2008;49:2870-2877. [PubMed: 18390636]

28. Evanko SP, Angello JC, Wight TN. Formation of hyaluronan- and versican-rich pericellular matrix is required for proliferation and migration of vascular smooth muscle cells. Arterioscler Thromb Vasc Biol 1999;19:1004-1013. [PubMed: 10195929]

29. Sćepanović OR, Fitzmaurice M, Gardecki JA, et al. Detection of morphological markers of vulnerable atherosclerotic plaque using multimodal spectroscopy. J Biomed Opt 2006;11 021007.

29. Jo JA, Fang Q, Papaioannou T, Qiao JH, et al. Diagnosis of vulnerable atherosclerotic plaques by time-resolved fluorescence spectroscopy and ultrasound imaging. Conf Proc IEEE Eng Med Biol Soc 2006;1:2663-2666. [PubMed: 17946129]

30. Caplan JD, Waxman S, Nesto RW, Muller JE. Near-infrared spectroscopy for the detection of vulnerable coronary artery plaques. J Am Coll Cardiol 2006;47:C92-C96. [PubMed: 16631516] 

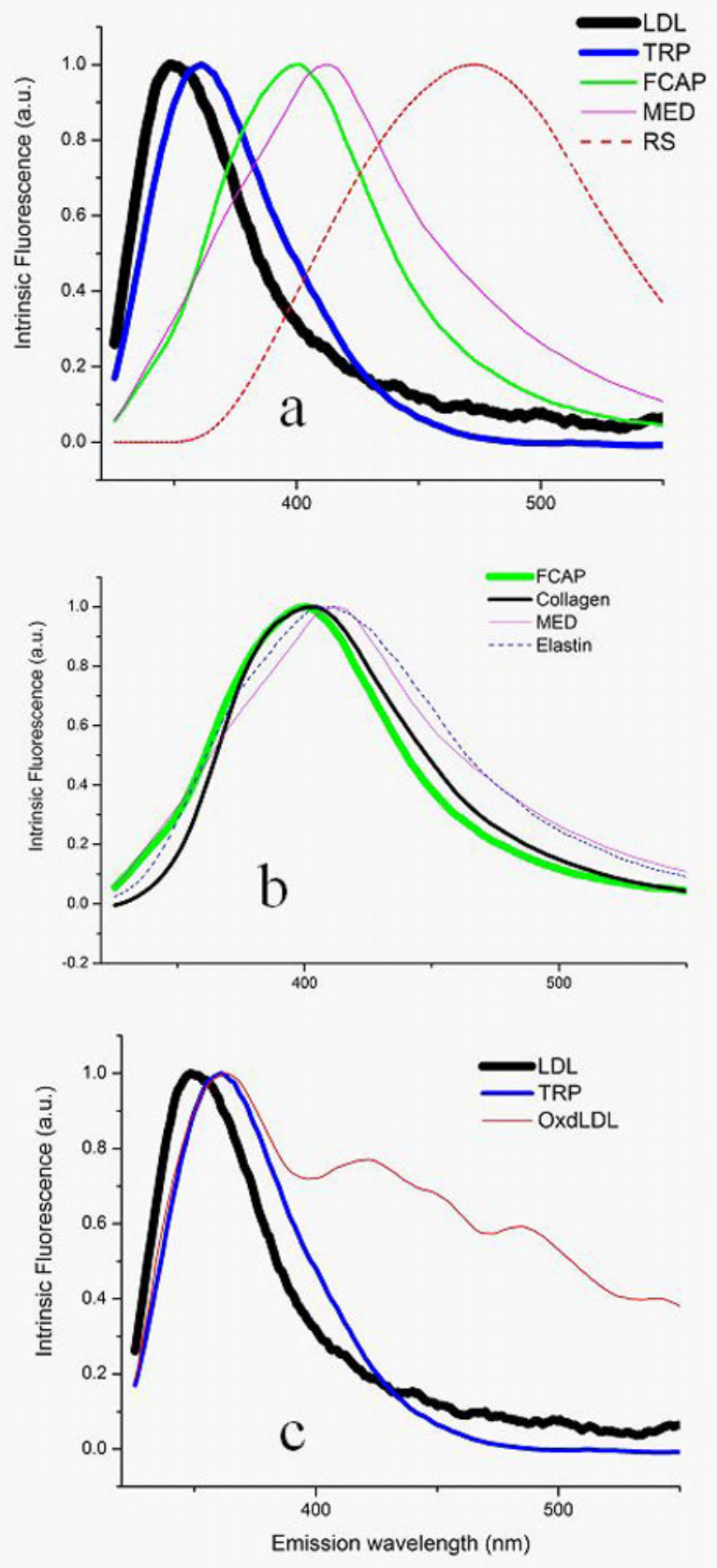

Figure 1.

Intrinsic fluorescence basis spectra at $308 \mathrm{~nm}$ excitation (a). Comparison between FCAP (fibrous cap spectrum) and MED (media spectrum) versus collagen and elastin spectra (b). Intrinsic fluorescence spectra at $308 \mathrm{~nm}$ excitation wavelength of low-density lipoprotein (LDL), tryptophan, and oxidized low-density lipoprotein (OxdLDL) (c). 

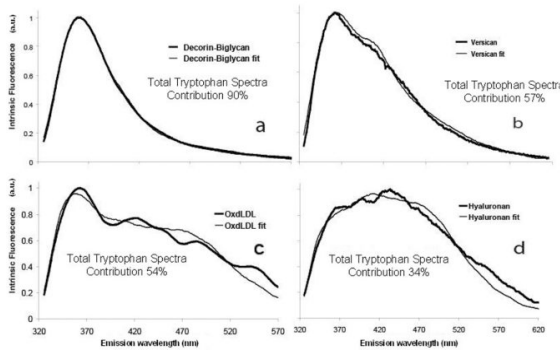

Figure 2.

Spectra and fit models of oxidized low-density lipoprotein (OxdLDL), decorin-biglycan, versican and hyaluronan. 


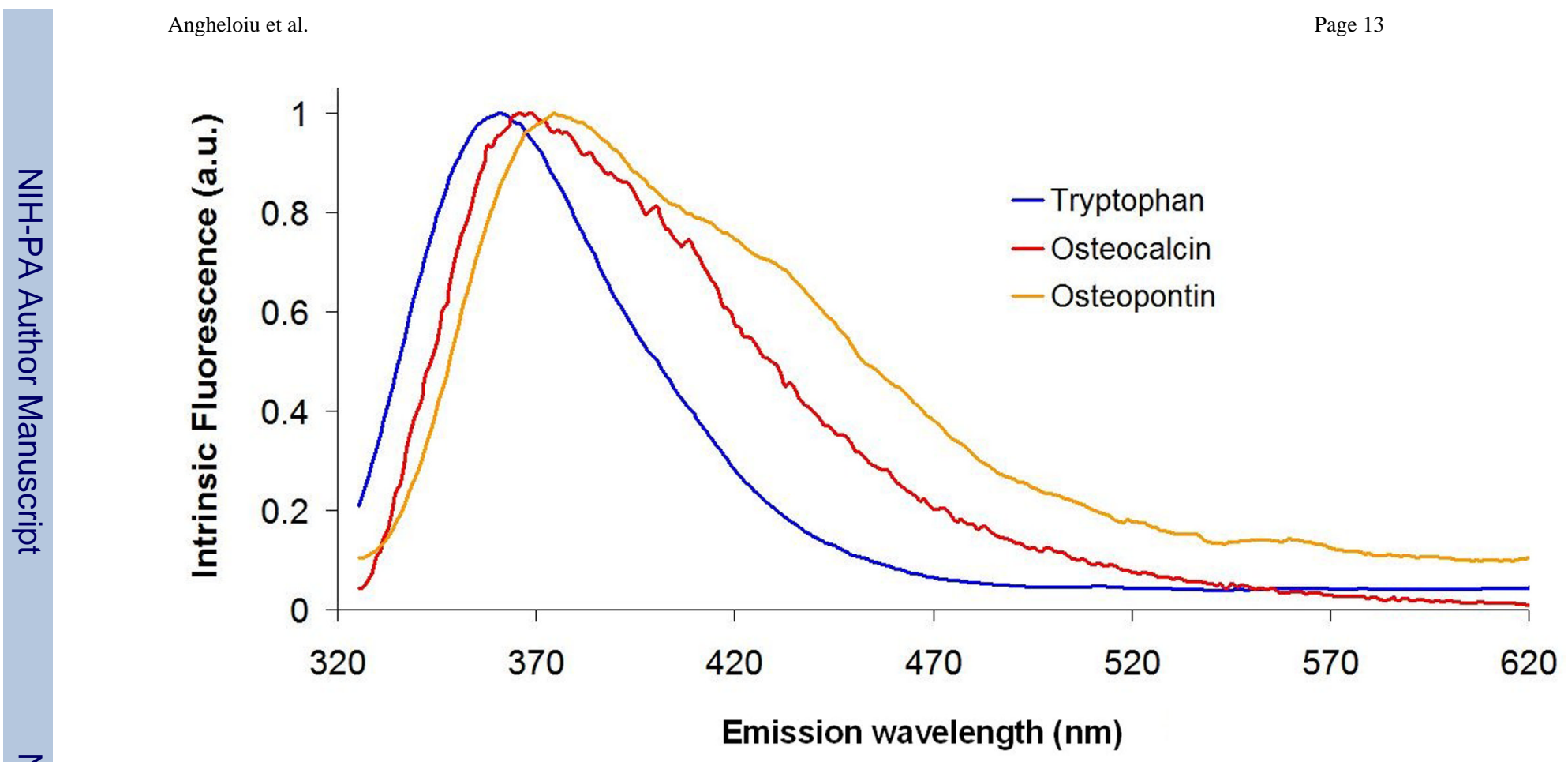

Figure 3.

Spectra of osteocalcin and osteopontin compared to the tryptophan spectrum. 


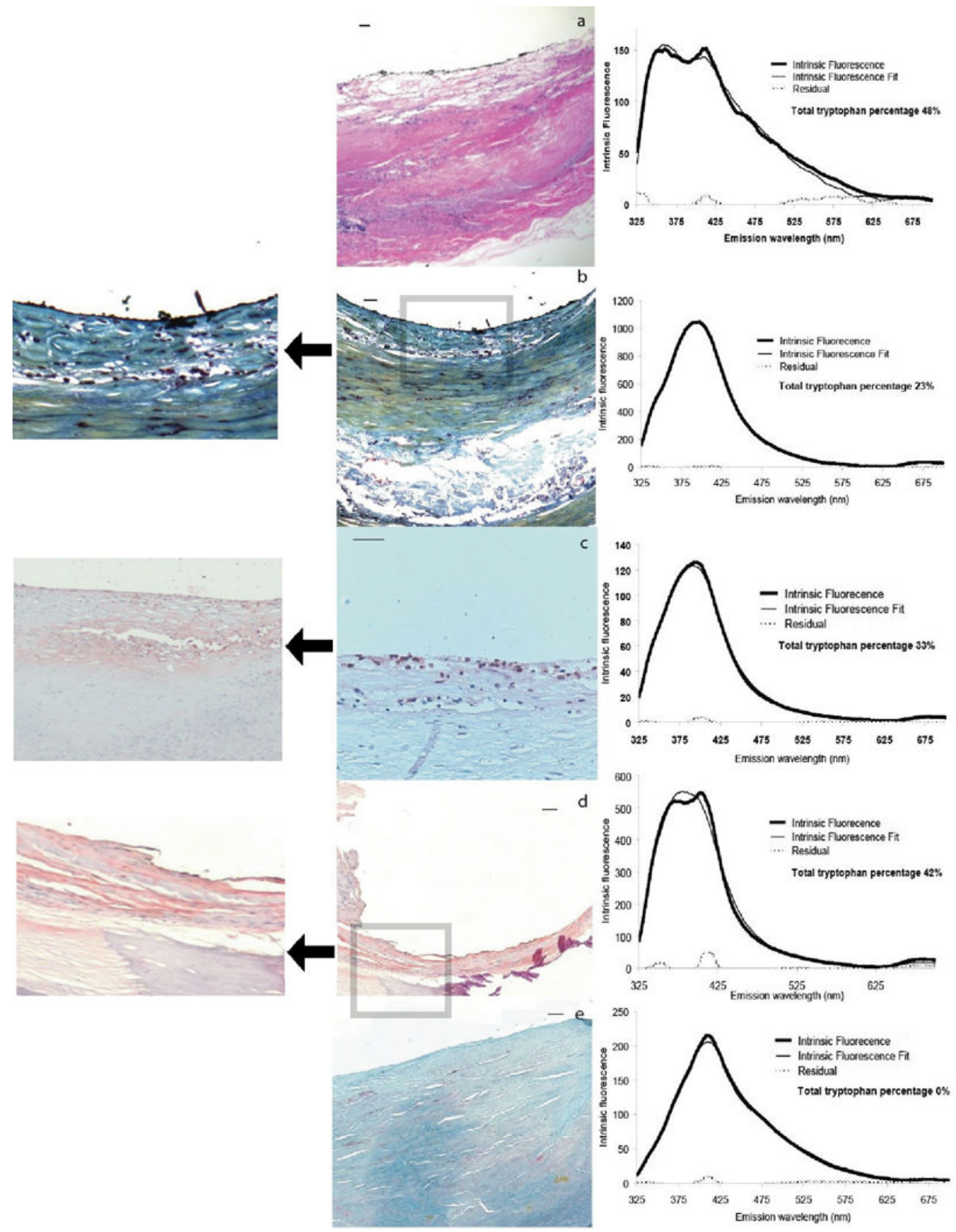

Figure 4.

Transversal sections of five human coronary specimens and their corresponding intrinsic fluorescence spectra and fit models at $308 \mathrm{~nm}$ : a) fibrous cap atheroma with high content of SFCs (superficial foam cells) and lymphocytic infiltration of intima and media (hematoxylin-eosin, x10), accompanied by proteoglycan accumulation in the superficial layers (Movat's pentachrome, not shown); b) fibrous cap atheroma with SFCs accompanied by proteoglycans in the top $50 \mu \mathrm{m}$ (Movat's pentachrome, blue coloration for proteoglycans, $\mathrm{x} 10$, with a zoom-in in the left panel for better illustration of SFCs); c) pathological intimal thickening with SFCs accompanied by proteoglycans (Movat's pentachrome, not shown) and versican accumulations (anti-versican antisera staining, red-brown coloration for 
versican, $\mathrm{x} 20$, with a zoom-out in the left panel for better illustration of anti-versican staining); d) calcified fibrous cap atheroma with intense accumulations of proteoglycans (Movat's pentachrome, not shown), versican (anti-versican antisera, not shown) and hyaluronan in the top $50 \mu \mathrm{m}$, accompanied by lymphocytic infiltration, in the absence of SFCs (hyaluronan binding protein staining, red-brown coloration for hyaluronan, $\mathrm{x} 10$, with a zoom-in in the left panel for better illustration of anti-hyaluronan staining); e) atherosclerotic specimen with no SFCs or proteoglycan accumulations (Movat's pentachrome, $\mathrm{x} 10)$. Solid bars indicate $50 \mu \mathrm{m}$ length. 


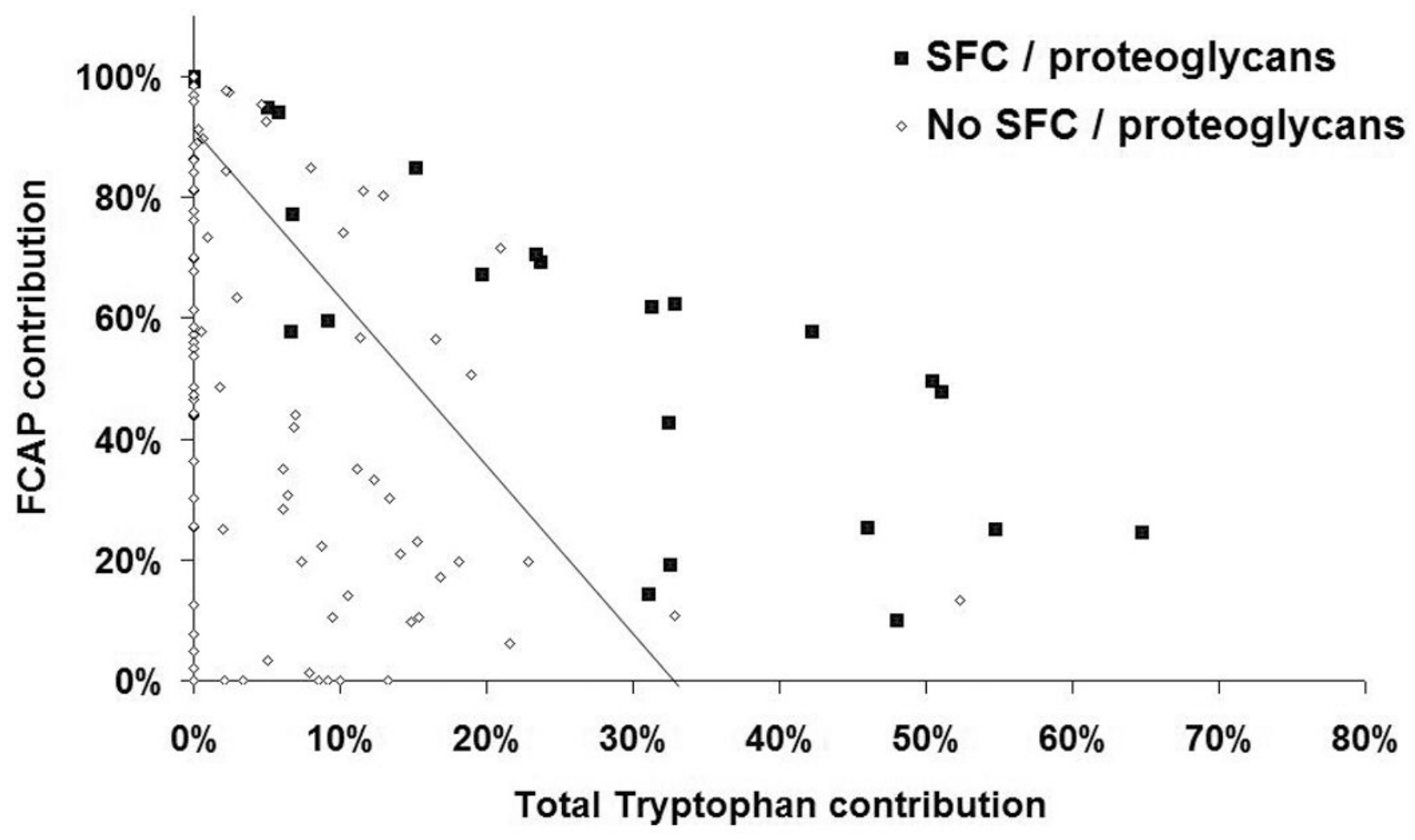

Figure 5.

Binary diagnostic algorithm for identification of specimens with SFCs (superficial foam cells) and/or proteoglycans. 